\title{
Denervation of gastroepiploic artery graft can reduce vasospasm
}

\author{
Yasutaka Yokoyama, MD, ${ }^{\text {a }}$ Satoshi Matsushita, $\mathrm{MD}, \mathrm{PhD},{ }^{\mathrm{a}}$ Takafumi Iesaki, $\mathrm{MD}, \mathrm{PhD},{ }^{\mathrm{b}}$ \\ Taira Yamamoto, MD, PhD, ${ }^{\text {a }}$ Hirotaka Inaba, MD, PhD, ${ }^{a}$ Takao Okada, MD, PhD, ${ }^{\mathrm{b}}$ and \\ Atsushi Amano, MD, $\mathrm{PhD}^{\mathrm{a}}$
}

\begin{abstract}
Background: The right gastroepiploic artery is useful as an in situ arterial graft for coronary artery bypass grafting. However, the gastroepiploic artery is more likely to cause vasospasms compared with the internal thoracic artery. We hypothesized that the cause of the spasms is the stimulation of the periarterial sympathetic nerve, because the gastroepiploic artery is classified as a muscular artery. In this study, we examined whether the spasm is reduced by removing the periarterial sympathetic nerve.
\end{abstract}

\begin{abstract}
Methods: Unused parts of the gastroepiploic artery were obtained from patients who underwent coronary artery bypass grafting. The vessel was cut into 2 segments, and they were assigned to control $(\mathrm{N}+)$ and denervation $(\mathrm{N}-)$ groups. The periarterial nerve was microscopically removed from the vessels of the $\mathrm{N}-$ group. The vessels in both groups were investigated by hematoxylin-eosin or immunohistochemical staining, and they were stimulated by electrical field stimulation with serial frequency for isometric tension measurement.
\end{abstract}

Results: Histologic analyses revealed that periarterial connective tissues including neuropeptide $\mathrm{Y}$ were removed to expose the external elastic membrane in the $\mathrm{N}-$ vessel, whereas they were preserved in $\mathrm{N}+$. The mean contraction by electrical field stimulation with serial frequency was consistently lower in $\mathrm{N}-$ than in $\mathrm{N}+(P<.05$ at 20 and $50 \mathrm{~Hz} ; \mathrm{n}=8$ each $)$. Endothelium-dependent relaxation and contractile function of the smooth muscle were similar in both groups.

Conclusions: The removal of the periarterial sympathetic nerve from the human gastroepiploic artery reduced vascular contraction, elicited by peripheral nerve stimulation, without disturbing endothelial and smooth muscle contractile functions. This reduction may contribute to the prevention of vasospasms. (J Thorac Cardiovasc Surg 2014;147:951-5)

The use of the right gastroepiploic artery (GEA) in coronary artery bypass grafting (CABG) was first reported in $1987 .{ }^{1,2}$ The GEA has the ability to supply blood to the posterolateral wall of the heart. Because it is an in situ arterial graft that obtains direct blood flow from systemic circulation, the use of the GEA prevents cerebral infarctions caused by operating on the ascending aorta. ${ }^{3}$ In case of a multivessel disease, complete revascularization has been shown to improve long-term survival. ${ }^{4}$ According to a recent report, $\mathrm{CABG}$ using multiple arterial grafts shows better late survival than CABG with left internal thoracic artery (ITA) to left anterior descending artery bypass and additional saphenous vein grafting. ${ }^{5}$ This indicates the advantage of multiple arterial conduits for complete revascularization. With increased prevalence of

\footnotetext{
From the Department of Cardiovascular Surgery, ${ }^{a}$ Juntendo University School of Medicine, Tokyo, Japan; and Department of Physiology, ${ }^{b}$ Juntendo University Faculty of Medicine, Tokyo, Japan.

Disclosures: Authors have nothing to disclose with regard to commercial support.

Received for publication Nov 13, 2012; revisions received Jan 6, 2013; accepted for publication Feb 12, 2013; available ahead of print March 13, 2013.

Address for reprints: Satoshi Matsushita, MD, PhD, Juntendo University, School of

Medicine, Cardiovascular Surgery, 2-1-1, Hongo, Bunkyo-ku, Tokyo, N/A 113-

8421 Japan (E-mail: saty-m@juntendo.ac.jp).

$0022-5223 / \$ 36.00$

Copyright (c) 2014 by The American Association for Thoracic Surgery

http://dx.doi.org/10.1016/j.jtcvs.2013.02.022
}

arteriosclerosis-causing diseases such as diabetes, the number of patients with multivessel coronary artery disease is increasing. Thus, there will be a growing demand for GEA.

However, the GEA grafts develop vasospasms more frequently than the ITA grafts. A vasospasm can result in postoperative shock and decreased long-term patency, ${ }^{6-8}$ and is known to be triggered by endothelial and autonomic nervous dysfunction..$^{9,10}$ According to a study of canine femoral arteries, which is classified as an elastic artery, vascular tonus effect of the sympathetic nerve is superior than the effect of an endothelial function. ${ }^{11}$ Because the GEA is a muscular artery that adjusts blood flow by the periarterial sympathetic nerve (PSN), there should be a greater effect of the periarterial nerve to its vascular contraction. This corresponds with the reports that vasospasm occurs more frequently in the GEA than in the ITA, which is classified as an elastic artery. ${ }^{12}$ In this study, we investigate whether removal of the PSN can reduce vasospasm using immunohistochemical and physiologic analyses.

\section{MATERIALS AND METHODS \\ Human Sample Collections}

Patients who underwent CABG using GEA in Juntendo University Hospital were included in this study. Handling of human samples followed the Declaration of Helsinki. This study was approved by the Human Ethics Committee of Juntendo University Hospital, and written informed consent was obtained from each patient. 


$$
\begin{aligned}
& \text { Abbreviations and Acronyms } \\
& \text { CABG }=\text { coronary artery bypass grafting } \\
& \text { EFS }=\text { electrical field stimulation } \\
& \text { GEA }=\text { gastroepiploic artery } \\
& \text { H\&E }=\text { hematoxylin-eosin } \\
& \text { ITA }=\text { internal thoracic artery } \\
& \text { NPY }=\text { neuropeptide Y } \\
& \text { PSN }=\text { periarterial sympathetic nerve } \\
& \text { TTX }=\text { tetrodotoxin }
\end{aligned}
$$

\section{Experimental Protocols}

Tissue preparation. Immediately after dissection, the GEA sample was transferred to the laboratory in a cold $\left(4^{\circ} \mathrm{C}\right)$ HEPES buffered $(25$ $\mathrm{mmol} / \mathrm{L}$ of HEPES, pH 7.4) Krebs bicarbonate solution containing the following: $118 \mathrm{NaCl} \mathrm{mmol} / \mathrm{L}, 4.7 \mathrm{KCl} \mathrm{mmol} / \mathrm{L}, 1.5 \mathrm{CaCl}_{2} \mathrm{mmol} / \mathrm{L}, 25$ $\mathrm{NaHCO}_{3} \mathrm{mmol} / \mathrm{L}, 1.1 \mathrm{MgSO}_{4} \mathrm{mmol} / \mathrm{L}, 1.2 \mathrm{KH}_{2} \mathrm{PO}_{4} \mathrm{mmol} / \mathrm{L}$, and 5.6 glucose $\mathrm{mmol} / \mathrm{L}$. All samples for physiologic study were investigated on the day of the collection. For the experiments, we used $2 \mathrm{~cm}$ from the proximal side of each vessel. Surrounding connective tissue and adipose tissue were removed using a pair of tweezers and scissors under the microscope, and the vessel was cut into 2 segments. One segment was used as control group $(\mathrm{N}+)$, and the other segment was used as the denervation group $(\mathrm{N}-)$. Connective tissue around the vessel of the denervation group was subsequently trimmed to the layer of external elastic membrane to remove periarterial nerves. After trimming, vessels in both groups were cut into 3 segments, with each segment measuring approximately $3 \mathrm{~mm}$. Two segments of the three in each group were used for isometric tension measurement, and the remaining segment was used for histologic study. Histologic study consisted of hematoxylin-eosin (H\&E) staining and immunohistochemical staining, and they were performed using frozen-section and whole mount of the vessel rings.

Hematoxylin-eosin staining. The frozen section was prepared by immersing the vessel rings in $4 \%$ paraformaldehyde for 30 minutes and then dehydrated by sucrose solutions of increasing concentration of $10 \%, 15 \%$, and $20 \%$. These rings were embedded in optimal cutting temperature compound (Sakura, Tokyo, Japan) and stored at $-80^{\circ} \mathrm{C}$. The frozen block was sectioned at $20 \mu \mathrm{m}$ using a cryomicrotome (Leica, Wetzlar, Germany) for H\&E staining. H\&E staining was applied to the sections for 15 minutes. After washing with water, the sections were immersed in $95 \%$ alcohol for 30 seconds and in eosin (Sigma-Aldrich, St Louis, Mo) for 60 seconds.

Immunohistochemical staining. Two axes (short and long axes) of immunostaining were performed. The short-axis slices were prepared using the frozen block as described earlier. The frozen tissue was sectioned at $5 \mu \mathrm{m}$ using a cryomicrotome and immersed in $0.5 \%$ Triton X-100 overnight. After blocking with $2 \%$ of goat serum (Sigma-Aldrich) for 30 minutes, the section was incubated with anti-neuropeptide Y (NPY) antibody (Enzo Life Sciences, Inc, Tokyo, Japan) at a dilution of 1:50 for 72 hours at $4^{\circ} \mathrm{C}$. F-actin was costained using rhodamine-phalloidin (Sigma-Aldrich) at a dilution of 1:200 for 1 hour at room temperature. After incubating with secondary antibodies (Alexa Fluor 488; Invitrogen, Tokyo, Japan) for 60 minutes, the section was observed under a fluorescence microscope (Keyence, Osaka, Japan).

In long-axis immunostaining, the whole mount vessel was used. The vessel was immersed in Zamboni solution (Sigma-Aldrich) for 48 hours and treated with $0.5 \%$ Triton X-100 overnight. After blocking with $1 \%$ of goat serum for 60 minutes, the vessel was incubated with anti-NPY antibody at a dilution of 1:50 for 72 hours at $4^{\circ} \mathrm{C}$. After incubating with secondary antibodies (Alexa Fluor 488) for 60 minutes, the vessel was observed with a confocal laser-scanning microscope (Leica).
Isometric tension measurement. Two vessel rings were prepared from each group. A total of 4 vessel rings were mounted on Magnus electrode wire hooks (Unique Medical, Tokyo, Japan) that were connected to force transducers (Nihon Kohden, Tokyo, Japan). Electrical field stimulation (EFS) was provided by an electrical stimulator (Dia Medical System, Tokyo, Japan), and the changes in isometric force were recorded by a polygraphic recording system (Rikadenki, Tokyo, Japan). The vessel rings were incubated in individually thermostated $\left(37^{\circ} \mathrm{C}\right) 10$-mL Magnus baths for 60 minutes at an optimal passive tension of $4 \mathrm{~g}$ in Krebs bicarbonate buffer gassed with $95 \% \mathrm{O}_{2}-5 \% \mathrm{CO}_{2}$. Vascular contraction of each vessel caused by high-KCL (high $\mathrm{K}^{+}$) Krebs in which $\mathrm{NaCl}$ was replaced by $\mathrm{KCl}(\mathrm{KCl}$ : $122.7 \mathrm{mmol} / \mathrm{L}$ ) was defined as maximum contraction $(100 \%)$.

Optimal voltage of EFS for each vessel was defined at the volt when the vascular contraction of $\mathrm{N}+$ occurred by increasing the voltage from 1 to 20 volts $(\mathrm{V})$ with square-wave pulses $(2.0 \mathrm{~ms}$ of duration) and $1 \mathrm{~Hz}$ frequency. After optimal voltage was obtained, vascular contraction was recorded with stimulate frequency of $1,2,5,10,20$, and $50 \mathrm{~Hz}$. The stimulation was applied for 30 seconds, and the vessels were stabilized until vascular contraction returned to baseline. The vessel rings that were not contracted by high $\mathrm{K}^{+}$Krebs or that were in vasospasm more than 6 hours were excluded from the study.

\section{Statistical Analysis}

The EFS data were evaluated using LabChart 7 software (AD Instruments, Nagoya, Japan), and the results were expressed as the means \pm standard error of the mean. The Student $t$ test was used to compare the data.

\section{RESULTS \\ Histologic Analyses}

$\mathrm{H} \& \mathrm{E}$ staining revealed that the periarterial tissues outside the external elastic membrane were maintained in $\mathrm{N}+$ vessel, whereas they were removed in $\mathrm{N}-$. Moreover, smooth muscle and endothelial cells were observed inside the external elastic membrane in both groups. These results showed that even after successful denervation, smooth muscle and endothelial cells were not injured by trimming (Figure 1, $A$ and $B$ ).

Double immunostaining of short-axis vessels was performed using NPY as a marker for sympathetic nerve and F-actin as a marker for smooth muscle. The NPY signals were detected around the external smooth muscle of $\mathrm{N}+$, and the signals were substantially reduced around the external smooth muscle of $\mathrm{N}-$ (Figure $1, C$ and $D$ ). Immunostaining of long-axis vessels with NPY as a marker for sympathetic nerve was performed. The NPY signals that formed a network around the vessel of $\mathrm{N}+$ had nearly disappeared in the vessel of $\mathrm{N}-($ Figure $1, E$ and $F$ ).

\section{Isometric Tension Measurement}

GEA contraction data from 8 cases are analyzed in Figure 2. Optimal volts for EFS in each vessel were $8 \mathrm{~V}$ in 4 cases, $10 \mathrm{~V}$ in 3 cases, and $20 \mathrm{~V}$ in 1 case. The mean contraction by EFS was consistently lower in $\mathrm{N}-$ than in $\mathrm{N}+: 2.5 \% \pm 1.5 \%, 4.7 \% \pm 2.8 \%, 9.9 \% \pm 4.6 \%$, $19.5 \% \pm 7.3 \%, 21.3 \% \pm 7.2 \%$, and $23.1 \% \pm 5.1 \%$ versus $7.0 \% \pm 3.9 \%, \quad 12.6 \% \pm 7.3 \%, \quad 22.5 \% \pm 9.5 \%$, $32.7 \% \pm 8.3 \%, 43.5 \% \pm 4.9 \%$, and $44.9 \% \pm 5.2 \%$ at $1,2,5,10,20$, and $50 \mathrm{~Hz}$, respectively $(P<.05$ at 20 and $50 \mathrm{~Hz})$. 

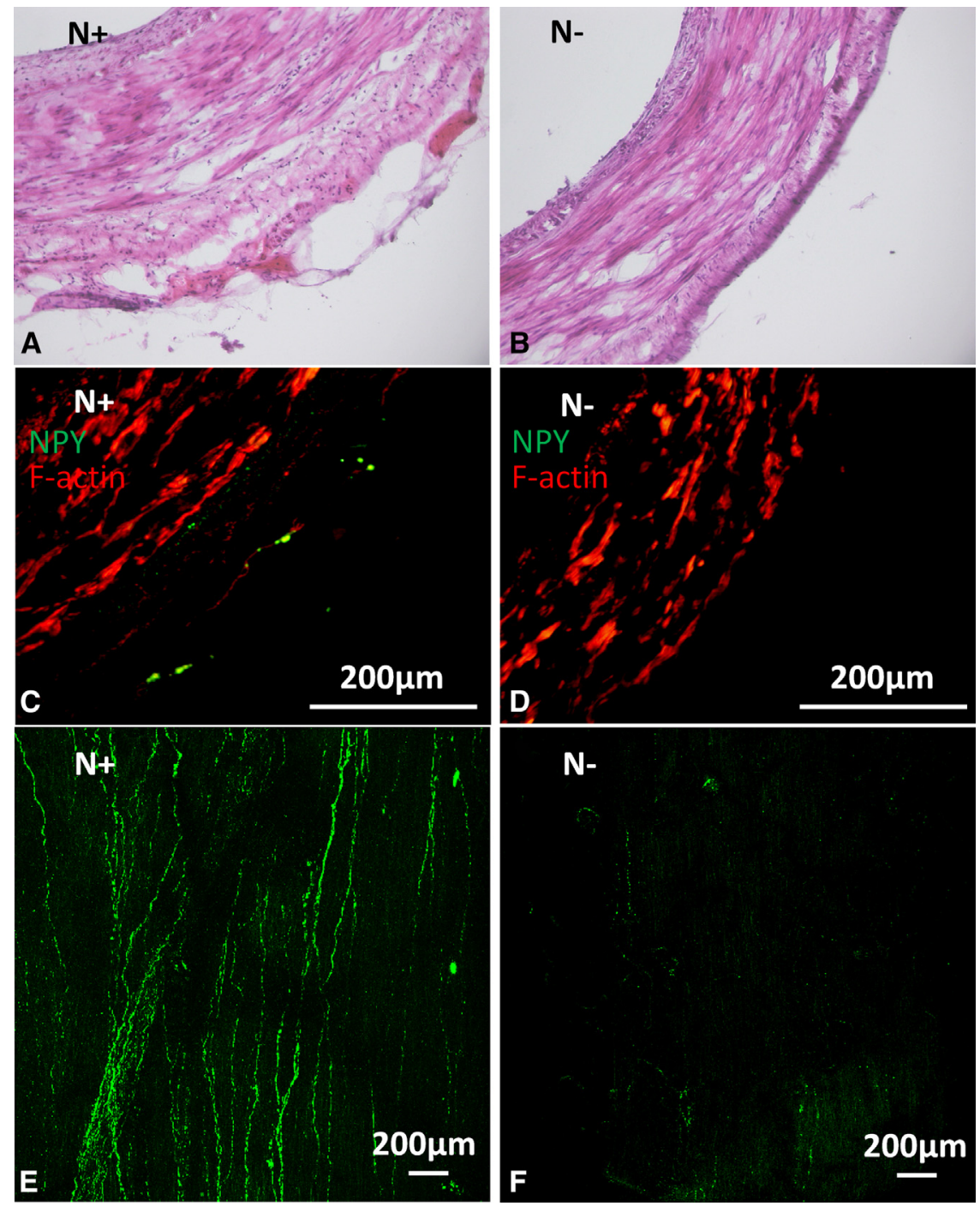

FIGURE 1. Histology of gastroepiploic artery PSN plexus. A and B, H\&E staining of a short-axis vessel. Periarterial tissues were outside the muscle layer of $\mathrm{N}+$. However, periarterial tissues were removed outside the muscle layer of $\mathrm{N}-$. $\mathrm{C}$ and $\mathrm{D}$, Double histochemical staining of a short-axis vessel for NPY and F-actin. NPY signal appeared outside the F-actin of N+. However, the NPY signal was removed outside the F-actin of N-. E and F, Histochemical staining of a long-axis vessel for NPY. NPY signal was observed to build a network around the vessel of N+; however, NPY signals showed that the network around the vessel of $\mathrm{N}-$ had nearly disappeared. NPY, Neuropeptide $\mathrm{Y}$.

Frequency-dependent vascular contraction was inhibited by tetrodotoxin (TTX), a voltage-sensitive sodium channel blocker that blocks the release of neurotransmitter from the nerve terminals. The contraction was almost eliminated in the presence of TTX: $0.0 \% \pm 0.0 \%, 0.3 \% \pm 0.1 \%$, $1.1 \% \pm 0.3 \%, 2.6 \% \pm 0.7 \%, 5.7 \% \pm 1.7 \%$, and $10.8 \% \pm 4.0 \%$ at $1,2,5,10,20$, and $50 \mathrm{~Hz}$, respectively $(P<.05$ from 5 to $50 \mathrm{~Hz}$, Figure 2, lower line). This result indicated that the vascular contraction induced by EFS was evoked via the depolarization of nerve terminals instead of a direct stimulation of the vascular smooth muscle.

The vessel rings were initially contracted with noradrenaline $(1 \mu \mathrm{mol} / \mathrm{L})$. Once a steady-state level of contraction was observed, acetylcholine $(1 \mathrm{nmol} / \mathrm{L}-10 \mu \mathrm{mol} / \mathrm{L})$ was applied cumulatively to evaluate the endotheliumdependent relaxation. There was no significant difference in endothelium-dependent vascular relaxation between $\mathrm{N}+$ and $\mathrm{N}-$. These results indicated that endothelial functions were not injured by trimming (Figure 3 ).

To determine whether the smooth muscle contractile function was sustained after harvest, maximum contractile force induced by high $\mathrm{K}^{+}$was obtained at the beginning and end of each experiment. There was no significant difference between the 2 groups at the beginning or end of the experiment (Figure 4). Furthermore, each vessel did not show a significant change in the value of maximum contractile force at the beginning and end of the experiment. These results indicated that contractile function of smooth muscle 


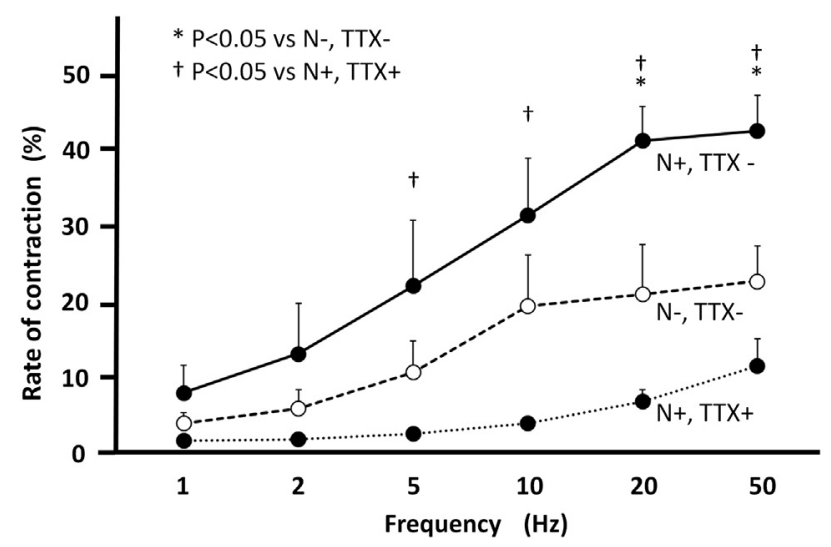

FIGURE 2. Gastroepiploic artery contraction to electrical field stimulation at frequencies from 1 to $50 \mathrm{~Hz}$. Contraction in $\mathrm{N}-$ was lower than in $\mathrm{N}+$ at all frequencies in the absence of TTX. Vascular contraction was almost eliminated in the presence of TTX, indicating that the contraction was evoked by the periarterial nerve plexus $(\mathrm{N}=8$, each). $\mathrm{N}+$, Gastroepiploic artery rings with periarterial nerve plexus; $\mathrm{N}-$, gastroepiploic artery rings without periarterial nerve plexus; TTX + , with $1 \mu \mathrm{mol} / \mathrm{L}$ TTX, TTX-, without $1 \mu \mathrm{mol} / \mathrm{L}$ TTX. TTX, Tetrodotoxin.

was not impaired by trimming or other experimental protocols.

\section{DISCUSSION}

This study revealed that vascular contraction caused by EFS in human GEA was reduced when its periarterial nerve was removed. Although the periarterial nerve was mostly removed by manual trimming, smooth muscle and endothelial function were preserved. Many studies have investigated the vascular contraction of the blood vessel preparations using periarterial nerve stimulations. ${ }^{13-17}$ However, we believe the present study is the first to investigate the vascular contraction of a blood vessel whose periarterial nerve was mechanically removed.

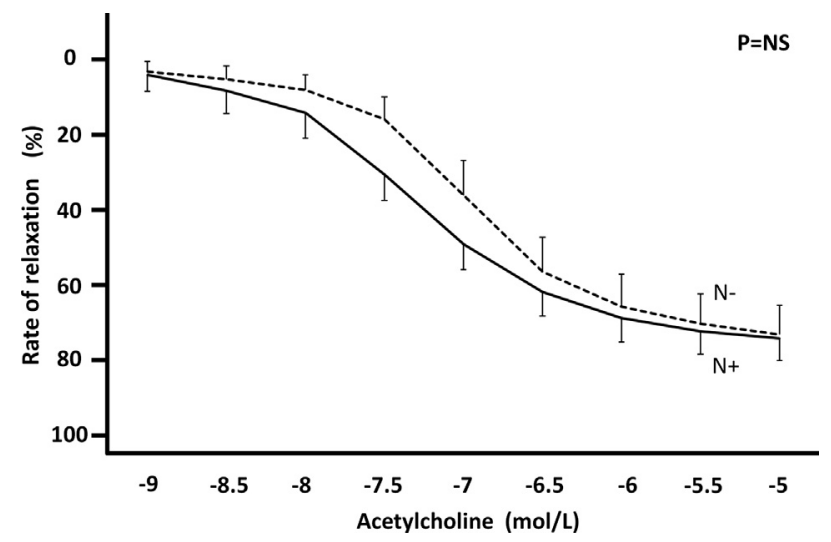

FIGURE 3. Relaxation of noradrenaline-precontracted gastroepiploic artery to acetylcholine. Relaxation was similar in both groups, indicating the endothelial function was not injured by trimming $(\mathrm{N}=8$, each). $\mathrm{N}+$, Gastroepiploic artery rings with periarterial nerve plexus; $\mathrm{N}-$, gastroepiploic artery rings without periarterial nerve plexus. $N S$, Not significant.

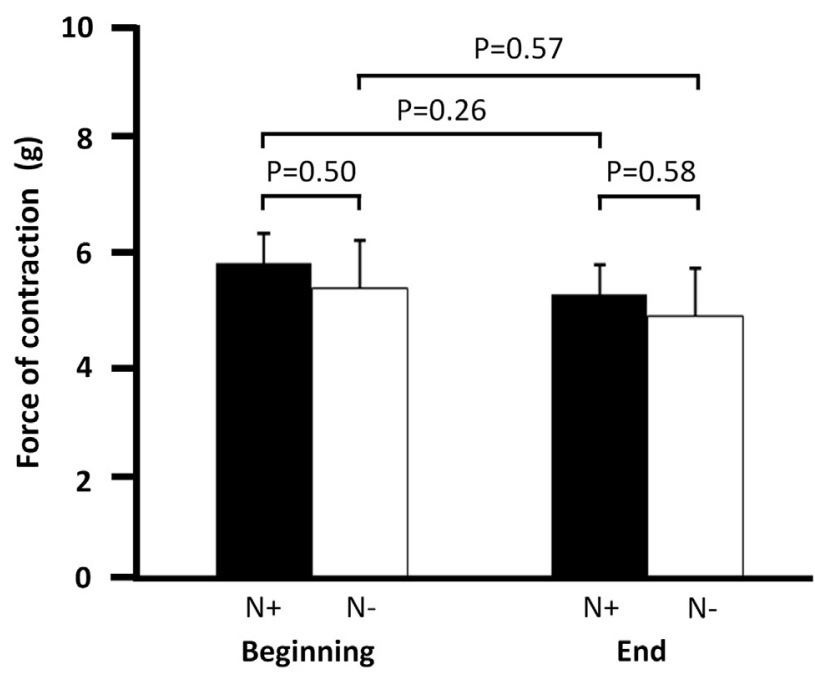

FIGURE 4. Gastroepiploic artery contraction to high $\mathrm{K}^{+}$at the beginning and end of the experiment. Contraction force in both groups was similar at the beginning and end of each experiment. In addition, decline of contraction was unrelated to the time course, indicating that the contractile function of smooth muscle was not injured by trimming or time course ( $\mathrm{N}=6$, each). $\mathrm{N}+$, Gastroepiploic artery rings with periarterial nerve plexus; $\mathrm{N}-$, gastroepiploic artery rings without periarterial nerve plexus.

NPY and calcitonin gene-related peptide are popularly known as typical marker proteins of perivascular nerve. ${ }^{18}$ Among these proteins, NPY is thought to exist on adrenaline and to enhance its effect. ${ }^{19}$ Immunostaining of NPY was performed to confirm the successful removal of the PSN in this study. In the long-axis staining, a uniformly built network of NPY-containing nerve fibers was seen around the vessels of $\mathrm{N}+$, whereas the network of nerve fibers around the vessels of $\mathrm{N}-$ had nearly disappeared. As in the previous study, ${ }^{20}$ short-axis staining showed NPY signals in periarterial tissues outside the external elastic membrane of $\mathrm{N}+$. However, NPY signals were not seen outside the external elastic membrane of $\mathrm{N}-$ in this study, and yet smooth muscle and endothelial cells inside the external elastic membrane were intact as in $\mathrm{N}+$.

An imbalance in the autonomic nervous system is reported to be one of the mechanisms of vasospasm. ${ }^{21}$ Under normal condition, the sympathetic nerve activity releases a vasoconstrictor substance (eg, noradrenaline) to bind to adrenergic receptors in the smooth muscle, resulting in smooth muscle contraction. ${ }^{22}$ The myosin light-chain phosphatase is responsible for the release of vascular contraction. The interruption of the phosphatase pathway causes continuous vascular contraction, which is reported to be the main mechanism of vasospasm. ${ }^{23}$ This vasospasm triggers the terminal of sympathetic nervous system to secrete additional vasoconstrictor substance, such as noradrenaline, which continues to act on the smooth muscle. EFS is commonly performed for functional evaluation to assess PSN. ${ }^{13-17}$ The strength of EFS is determined by voltage, frequency, 
and duration. According to previous reports, the secretion of noradrenaline from the terminal of sympathetic nervous is dependent on frequency. ${ }^{14}$ Thus, only the frequency was altered in this study, whereas the voltage and the duration were kept unchanged. Although vascular contraction increased depending on the frequency in both groups, the contraction of $\mathrm{N}$ - was consistently lower than that of $\mathrm{N}+$ at all frequencies. Because of the removal of PSN and the elimination of the nerve network, there was a decrease in the secretion of noradrenaline from the terminal of sympathetic nerves. However, an increase in vascular contraction of $\mathrm{N}$ - was observed with increasing frequency. As the result of immunostaining shows, the PSN of N- was mostly but incompletely removed, and noradrenaline was secreted by the remaining nerve terminals. Furthermore, the vascular contraction was inhibited by TTX, a neuroleptic agent, in both groups. This proves that the vascular contraction to EFS in this study was caused by periarterial nerve stimulation.

Other factors associated with vasospasm are endothelial and smooth muscle function. Nitric oxide, which is produced from endothelial cells by acetylcholine stimulation, causes relaxation of the smooth muscle. ${ }^{9,10,20}$ Cumulative concentration of acetylcholine was added to evaluate the endothelium-dependent vascular relaxation in vessels precontracted with noradrenaline. As shown in the results section, endothelial function and contractile function of smooth muscle were similar in both groups. These results were consistent with the outcomes of the histologic investigation using immunostaining. Furthermore, vascular force development was not significantly decreased at the end of the experiment in both groups. These results show that contractile function of the smooth muscle was not injured during experimental protocols. Although human GEA was used and EFS was performed to simulate the in vivo condition, further studies, including layer analysis of the "skeletonized" vessels, are necessary to evaluate the efficacy in the clinical setting, such as prevention of perioperative vasospasm, long-term patency, and even improvement in survival.

\section{CONCLUSIONS}

The present study indicates that trimming of perivascular connective tissue could remove the periarterial nerve plexus while preserving endothelial and contractile function of the smooth muscle. Moreover, vascular contraction through the periarterial nerve plexus was reduced by denervation, suggesting that the removal of the periarterial nerve plexus inhibits the vasospasm.

The authors thank Hiromu Kawasaki (Okayama University), Narumi Hashikawa (Okayama University of Science), and Mitsuhiro Goda (Setsunan University) for providing excellent technical support in performing the immunohistochemical staining for PSN; Katsumi Miyahara (Juntendo University Division of biomedical Imaging research) for providing excellent technical support for a confocal laser-scanning microscope; and Yuko Kojima for correcting the English in this article.

\section{References}

1. Suma H, Fukumoto H, Takeuchi A. Coronary artery bypass grafting by utilizing in situ right gastroepiploic artery: basic study and clinical application. Ann Thorac Surg. 1987;44:394-7.

2. Pym J, Brown PM, Charrette EJ, Parker JO, West RO. Gastroepiploic-coronary anastomosis. A viable alternative bypass graft. J Thorac Cardiovasc Surg. 1987;94:256-9

3. Lev-Ran O, Braunstein R, Sharony R, Kramer A, Paz Y, Mohr R, et al. No-touch aorta off-pump coronary surgery: the effect on stroke. J Thorac Cardiovasc Surg. 2005; 129:307-13

4. Locker C, Schaff HV, Dearani JA, Joyce LD, Park SJ, Burkhart HM, et al. Multiple arterial grafts improve late survival of patients undergoing CABG: analysis of 8,622 patients with multivessel disease. Circulation. 2012;126:1023-30.

5. Jones EL, Weintraub WS. The importance of completeness of revascularization during long-term follow-up after coronary artery operations. J Thorac Cardiovasc Surg. 1996;112:227-37.

6. Suma H. Spasm of the gastroepiploic artery graft. Ann Thorac Surg. 1990;49: 168-9.

7. Cate CM, Gitter R, Jett K. Spasm of the gastroepiploic artery used for coronary artery bypass grafting. Am J Cardiol. 1996;77:1022-3.

8. Lorusso R, Crudeli E, Luc • F, De Cicco G, Vizzardi E, D’Aloia A, et al. Refractory spasm of coronary arteries and grafted conduits after isolated coronary artery bypass surgery. Ann Thorac Surg. 2012;93:545-51.

9. Kawano H, Ogawa $\mathrm{H}$. Endothelial function and coronary spastic angina. Intern Med. 2005;44:91-9.

10. Miwa K, Fujita M, Sasayama S. Recent insights into the mechanisms, predisposing factors, and racial differences of coronary vasospasm. Heart Vessels. 2005;20:1-7.

11. Funahashi S, Komori K, Itoh H, Okadome K, Sugimachi K. Effects of lumbar sympathectomy on the properties of both endothelium and smooth muscle cells of the canine femoral artery and autogenous vein grafts under poor runoff conditions. J Surg Res. 1996;64:184-9.

12. He GW, Yang CQ. Comparison among arterial grafts and coronary artery. An attempt at functional classification. J Thorac Cardiovasc Surg. 1995;109:707-15.

13. Fukui D, Yang XP, Chiba S. Neurogenic double-peaked vasoconstriction of human gastroepiploic artery is mediated by both alpha1- and alpha2-adrenoceptors. Br J Pharmacol. 2005;144:737-42.

14. del Campo L, Blanco-Rivero J, Balfagon G. Fenofibrate increases neuronal vasoconstrictor response in mesenteric arteries from diabetic rats: role of noradrenaline, neuronal nitric oxide and calcitonin gene-related peptide. Eur J Pharmacol. 2011;666:142-9.

15. Donoso MV, Miranda R, IrarrÁaval MJ, Huidobro-Toro JP. Neuropeptide Y is released from human mammary and radial vascular biopsies and is a functional modulator of sympathetic cotransmission. J Vasc Res. 2004;41:387-99.

16. Yang XP, Chiba S. Pharmacological analysis of the double peaked vasoconstrictor responses to periarterial electric stimulation. J Auton Pharmacol. 1998;18:343-7.

17. Ren LM, Nakane T, Chiba S. Characteristics of the responses of isolated and perfused canine splenic arteries to vasoactive substances and to periarterially electrical stimulation. Jpn J Pharmacol. 1994;64:19-25.

18. Hobara N, Yoshida N, Goda M, Yokomizo A, Kitamura Y, Sendou T, et al. Neurotrophic effect of hepatic growth factor (HGF) on reinnervation of perivascular calcitonin gene-related peptide (CGRP)-containing nerves following phenol-induced nerve injury in the rat mesenteric artery. J Pharmacol Sci. 2008;108:495-504.

19. Kasakov L, Ellis J, Kirkpatrick K, Milner P, Burnstock G. Direct evidence for concomitant release of noradrenaline, adenosine 5'-triphosphate and neuropeptide $\mathrm{Y}$ from sympathetic nerve supplying the guinea-pig vas deferens. J Auton Nerv Syst. 1988;22:75-82.

20. Deja MA, Gołba KS, Malinowski M, Woś S, Kolowca M, Biernat J, et al. Skeletonization of internal thoracic artery affects its innervation and reactivity. Eur J Cardiothorac Surg. 2005;28:551-7.

21. Sakata K, Yoshida H, Hoshino T, Kurata C. Sympathetic nerve activity in the spasm-induced coronary artery region is associated with disease activity of vasospastic angina. J Am Coll Cardiol. 1996;28:460-4.

22. Yasue H, Touyama M, Shimamoto M, Kato H, Tanaka S. Role of autonomic nervous system in the pathogenesis of Prinzmetal's variant form of angina. Circula tion. 1974;50:534-9.

23. Fukata Y, Amano M, Kaibuchi K. Rho-Rho-kinase pathway in smooth muscle contraction and cytoskeletal reorganization of non-muscle cells. Trends Pharma col Sci. 2001;22:32-9. 